\title{
RPAS integration in non-segregated airspace: Safety metrics for tactical planning
}

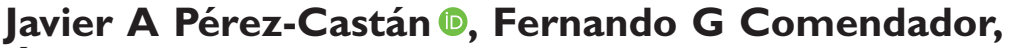 \\ Álvaro Rodriguez-Sanz $\mathbb{\oplus}$, Rosa M Arnaldo Valdés and \\ Gonzalo Agueda
}

\begin{abstract}
The integration of remotely piloted aircraft system in non-segregated airspace requires a significant effort and new methodologies to underway this challenge. This paper develops a methodology to assess the impact of remotely piloted aircraft system integration by applying safety metrics in tactical planning. This methodology builds five modules to simulate remotely piloted aircraft system introduction in a conventional-aircraft schedule: Base scenario, path modelling, conflict detection, temporary-blocking window and safety metrics. The safety metrics quantify the safety state of the operation by the number of conflicts, the conflict severity and the airway availability. This last safety metric represents a step forward in the decision-making process because it provides the airway risk-suitability to integrate remotely piloted aircraft system. Moreover, the temporary-blocking window underlies the airway availability metric. This concept provides temporary restrictions to the integration of remotely piloted aircraft system depending on the entry times of the conventional aircraft. Finally, this methodology is applied in an air traffic volume of the Spanish upper airspace. Different simulations were performed by introducing remotely piloted aircraft system covering every airway of the airspace. Results provided the temporary-blocking windows that specified the temporary restrictions to remotely piloted aircraft system introduction as a function of the airway flown by the conventional aircraft. Furthermore, the methodology appraised the airway availability characterising the airways depending on the risk impact by the remotely piloted aircraft system.
\end{abstract}

\section{Keywords}

Air transport, RPAS, safety metrics, tactical planning, temporary-blocking window

Date received: 21 February 2019; accepted: II June 2019

\section{Introduction}

The integration of remotely piloted aircraft systems (RPASs) into non-segregated airspace jointly with conventional aircraft is extremely relevant to the aviation community. The European Steering group plans to integrate RPAS in non-segregated airspace in 2025 horizon. ${ }^{1}$ Major efforts focused on developing regulation related to requisites and performances that must comply with these aircraft. ${ }^{2,3}$ Nonetheless, the vast majority of this regulation aims to RPAS for specific missions that do not respond to the necessities of systematic integration in airspace jointly with conventional aircraft. ${ }^{4,5}$ The scale of the problem is completely different because one assesses the integration of one specific RPAS in the air traffic management (ATM) system and the other analyses how the integration of RPAS affects the ATM system.

Therefore, it is required to analyse and plan a methodical RPAS integration with conventional aircraft in the ATM system. The major issue underlies that few works are evaluating the safety impact imposed by the RPAS integration jointly with conventional aircraft. A thorough literature review of the main works developed so far indicates that research is mainly focused on four areas: Conflict Detection and Resolution (CD\&R), third-party risk models, safety risk management and safety impact in non-segregated airspace. Notably, the authors highlight the literature research of Netjasov and Janic ${ }^{6}$ because they performed a thorough review of the different risk models.

Universidad Politécnica de Madrid, Pza. Cardenal Cisneros, Madrid, Spain

Corresponding author:

Javier A. Pérez-Castán, Universidad Politécnica de Madrid, 3 Madrid 28040, Spain.

Email: javier.perez.castan@upm.es 
CD\&R for conventional aircraft is an issue extensively analysed for many years. However, conflict analysis between RPAS and conventional aircraft is more recent. Because of the poor vertical performances of pioneering RPAS, CD\&R was based on horizontal manoeuvres. ${ }^{7,8}$ In this way, more mathematically complex solutions were carried out seeking the optimisation of CD\&R regarding time and fuel consumption. ${ }^{9-11}$ Currently, RPAS can comply with vertical performance requirements in the upper airspace. Therefore, the vertical conflict resolution implies a better use of the airspace as well as the improvement of Air Traffic Control (ATC) situational awareness. ${ }^{12}$ The aviation community is evaluating the optimisation of CD\&R from a different point of view: such as fuel consumption, ATC workload, capacity and so on. However, current challenges do not reside in the resolution of a singular conflict but the safe integration of RPAS in the air transport system.

Risk models for RPAS operation focus on thirdparty risk models. These risk models meet the solution to operate RPAS in low-altitude airspaces, mostly aircraft that overfly urban areas. These risk models aim to ascertain the risk of a pedestrian, ${ }^{13}$ a building ${ }^{14}$ or a mid-air collision ${ }^{15}$ due to an impact by the RPAS. Melnyk et al. ${ }^{16}$ performed an in-depth comparison of the different ground-risk parameters and prediction methods of impact area by different authors. The most complex study was carried out by Lum and Waggoner. ${ }^{17}$ The authors modelled the pedestrian impact and the probability of a mid-air collision based on the collision frequency of gas molecules for the whole National Airspace System (NAS). However, these researches tackle a specific need of an individual flight plan but not the methodical integration.

The third large area focuses on safety risk management for RPAS operations. This concept is a widespread topic, but its major goal is to define the operational requirements that one RPAS must fulfil to operate in non-segregated airspace safely. RománCordón et al. ${ }^{18}$ designed requirements for an on-ground station regarding design, interface and communications issues. Clothier et al. ${ }^{19,20}$ developed an airworthiness regulatory framework for RPAS. The proposed approach defined a methodical, objective and justifiable method for managing the risk associated with the diversity of RPAS and their potential operations. Following a similar approach, different studies $^{2,5,21,22}$ structured the safety case of RPAS operation, detailing the different elements and systems that must be required by RPAS. Particularly, JARUS developed the guidelines for the risk assessment methodology (SORA) to establish a sufficient level of confidence for a specific RPAS operation. ${ }^{23}$

The last research group deals with, perhaps, the biggest issue: the entire integration of RPAS in nonsegregated airspace. SORA can be a good baseline for this integration of RPAS; however, it focuses on specific RPAS operations from the point of view of the operator. One of the requirements imposed by EASA is that the RPAS integration cannot imply a decrease in the operational risk. ${ }^{3,24}$ Although there are different models to assess the operational risk in airspace, ${ }^{25-27}$ few of them analysed the impact of the RPAS integration. Pérez-Castán et al. ${ }^{28}$ applied a conflict-risk model performing simulations with RPAS in non-segregated airspace to determine the geographical restrictions to RPAS, in strategical planning. $\mathrm{Kim}^{29}$ assessed the conflict risk between small RPAS taking into account the position uncertainty of these aircraft. Jenie et al. ${ }^{30}$ performed Monte Carlo Simulations to include the effect of the variety of CD\&R systems in each aircraft. However, most of the efforts do not tackle the tactical planning and the temporary restrictions for the safe integration of RPAS.

The main goal of this paper is to develop a methodology and safety metrics that allow for the safe integration of RPAS in non-segregated airspace in tactical planning. To achieve that, the authors propose three safety metrics: number of conflicts, conflict severity and airway availability. These safety metrics characterise the situation of the airspace and allow for taking measures about the viability of RPAS introduction. A further goal is to determine temporary restrictions to the RPAS because, by calculating temporary-blocking windows (TBWs), it can be specified when the RPAS can or not pierce into the airspace. The remainder of the paper is as follows. The Methodology section describes the methodology that is constituted by five modules: base-scenario, path modelling, conflict detection, temporary-blocking window and safety metrics. The Case study section introduces the case study to validate the methodology by describing the scenario and the conventionalaircraft schedule. The Results section provides the results of safety metrics by integrating RPAS jointly with conventional aircraft in different situations, and the Discussion of results section discusses the results obtained. Lastly, the Conclusions section summarises the major conclusions of the research.

\section{Methodology}

This methodology aims to determine the viability of the safe integration of RPAS in tactical planning. The ATM process at large encompasses three planning phases: strategical, pre-tactical and tactical. The air traffic information evolves from the strategical (until one year before) to the tactical phase (the same day of the operation). The ATM process varies since the knowledge of a reference hour (based on air traffic flows) to an operational hour (based on specific information about the aircraft operations). Throughout this process, the Air Navigation Service Provider (ANSP) safely adjusts the flight plans balancing the demand and the capacity. 


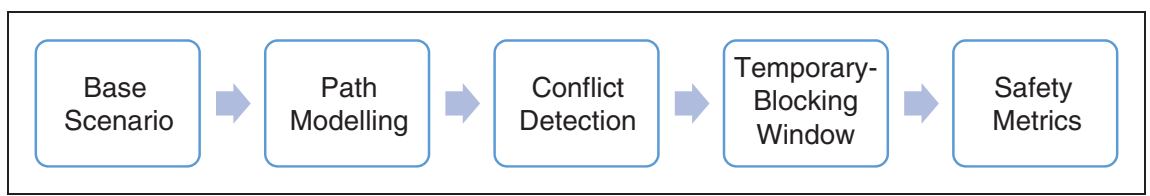

Figure I. Scheme of the process to integrate RPAS in tactical planning.

This methodology provides valuable information to the ANSP in tactical planning, adjusting a safe integration of RPAS. This goal is achieved by the identification of the temporary restrictions and the airway availability. In this way, the methodology provides the temporal periods in which the RPAS introduction does not generate conflicts. Therefore, the RPAS does not generate any disturbance with other aircraft. This methodology requires two sources of information: (a) geometrical information from the airspace morphology and (b) operational information of the air traffic. The development of the methodology follows a process that finalises with the calculation of three safety metrics. The following modules constitute this process:

1. Base scenario: this module provides the information of the airspace design and the aircraft schedule that is going to be considered.

2. Path modelling: this module generates the trajectories (conventional aircraft and RPAS) that constitute the aircraft one-hour schedule.

3. Conflict detection: this module evaluates the trajectory evolution and determines the violation of separation minima between aircraft. In this study, one conflict is defined as the situation where a separation minima infringement is going to happen.

4. Temporary-blocking window: this module calculates the TBWs that impose incompatibilities between conventional aircraft and RPAS. These TBWs are characterised by the entry time, the airways used and the ground speeds. With this information, the algorithm calculates the location and duration of time spans in which an aircraft cannot use an airway due to conflict generation.

5. Safety metrics: the last module calculates the safety metrics based on risk indicators that quantify the impact that will imply the integration of RPAS in tactical planning.

Figure 1 summarises the scheme of the methodology described above, and the following sections detail the modelling of the different modules.

\section{Base scenario}

The first module encompasses the information of the base scenario to apply the methodology. Each scenario is fixed airspace operated for different temporary schedules. The geometrical information is based on the morphology of the airspace: the airway indicator $(j)$

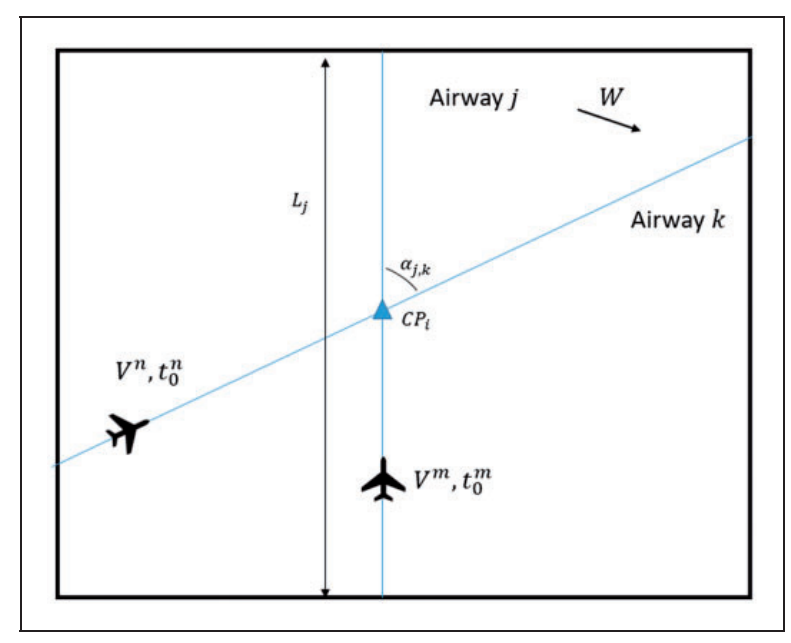

Figure 2. Scheme of a generic situation with an intersection.

and length of the airways $\left(L_{j}\right)$ and the geometry of the crossing points (the crossing-point indicator $C P_{i}$ and the crossing angle $\alpha_{j, k}$ ). Moreover, operational information of the air traffic is required. The main variables in a flight schedule are the ground speed for each aircraft $\left(V^{m}\right)$ and the entry time to the airspace $\left(t_{0}^{m}\right)$. Figure 2 shows a representation of a basic intersection of the airspace and the main variables.

\section{Path modelling}

The second module generates the trajectories of the aircraft that operate in the airspace. This study develops en-route flights uniquely, i.e. it is not considered flight level changes or climbing/descending performances (although they will be included in further work). The introduction of the aircraft climbing or descending does not imply any complexity except the development of new trajectories modifying the cinematic equations. ${ }^{31-33}$ Moreover, we do not consider uncertainty associated with the aircraft velocity or the positioning because it is not considered a probabilistic study of conflict risk.

Then, an aircraft $m$ defines its position by three coordinates: $X^{m}, Y^{m}$ and $Z^{m}$. The cinematic equations that build the trajectory as a function of the time $(t)$ are the following

$$
\begin{aligned}
& X^{m}(t)=X_{0}^{m}+\left(V_{x}^{m}-W_{x}\right) t \\
& Y^{m}(t)=Y_{0}^{m}+\left(V_{y}^{m}-W_{y}\right) t \\
& Z^{m}(t)=Z_{0}^{m}+\left(V_{z}^{m}-W_{y}\right) t
\end{aligned}
$$


where $X_{0}^{m}, Y_{0}^{m}$ and $Z_{0}^{m}$ are the initial positions of the aircraft $(t=0), V_{x}^{m}, V_{y}^{m}$ and $V_{z}^{m}$ are the speed projected in the axis $X^{m}$, $Y^{m}$ and $Z^{m}$, and $W_{x}$ and $W_{y}$ are the wind forces projected in the corresponding axis.

\section{Conflict detection}

The third module evaluates the evolution of the aircraft separation with the goal of detecting conflicts between them. The evaluation of the separation $\left(D^{m, n}(t)\right)$ must be performed between an aircraft pair $m, n$. This calculation is performed on the horizontal profile because both aircraft fly at the same Flight Level (FL). This allows knowing the instant of time and the minimum separation as a function of time

$$
D^{m, n}(t)=\sqrt{\left(X^{m}(t)-X^{n}(t)\right)^{2}+\left(Y^{m}(t)-Y^{n}(t)\right)^{2}}
$$

The condition of conflict is that the horizontal separation minimum $\left(S_{\min }\right)$ fulfills $D^{m, n}(t)<S_{\min }$. The horizontal separation minima in the upper airspace in Spain is 5 Nautical Miles (NM) ${ }^{34}$ The instants of time between the separation minima infringement will be crucial to calculate the conflict severity.

\section{Temporary-blocking window}

The fourth module calculates the TBW between an aircraft pair. The TBW is the time span associated with an aircraft pair flying intersecting airways in which if an aircraft pierces into the airspace in the corresponding TBW, they will conflict. The concept of TBWs is based on the blocking-area concept. A blocking-area is a geographical window that fulfils the following condition: if an aircraft is inside the blocking-area, another aircraft will suffer a block to its regular operation. The blocking-area concept has been developed previously in different scenarios ${ }^{35-38}$ focused on the implementation of restrictions between departures and arrivals in complex terminal airspaces. Therefore, this module is the most valuable of this research because it develops and applies the TBW concept for safety analysis of airspace design. TBWs allow for calculating temporary restrictions to the introduction of RPAS in non-segregated airspace. In turn, these temporary restrictions should be integrated into the calculation and design of the flightplan process for the RPAS operation. In this way, it is ensured that an aircraft that pierces into the airspace does not generate any conflict.

Figure 3 represents a scheme of the calculation of the TBW between two airways that intersects. In this figure, two aircraft $(m, n)$ operate through their respective airways $(j, k)$ and concur at the crossing point $C P_{i}$ with a crossing angle $\alpha_{j, k}$. The algorithm of the temporary-blocking window is defined to characterise the influence of the aircraft $m$ on the aircraft $n$ when the aircraft $m$ pierces into the airspace.

The conflict area is the circle centred at the crossing point and whose diameter is the critical section $d_{j, k}$. In this case, if one aircraft is inside the critical section, another aircraft (or RPAS) cannot be at the same time in the conflict area.

$$
d_{j, k}=\frac{2 S_{\min }}{\sin \alpha_{j, k}}
$$

Based on the position of the aircraft $m$, there are two critical situations regarding the initial or final points of the TBW. If the aircraft pierces into these two critical situations, a separation minima infringement will occur.

1. The initial critical situation occurs when the conventional aircraft is at the beginning of the conflict area, and the RPAS is leaving.

2. The final critical situation occurs when the conventional aircraft is leaving the conflict area, and the RPAS is entering.

The equations to build the location and the size of the TBWs depends on the aircraft velocities, the distance from the entry point of the aircraft $m$ to the crossing point $\left(d_{C P_{i}, j}\right)$ and the entry time $t_{0}^{m}$.

$$
\begin{aligned}
d_{B W}^{m, n} & =d_{j, k}+d_{e}-d_{f} \\
d_{e} & =d_{2} \frac{V^{n}}{V^{m}} ; \quad d_{f}=d_{1} \frac{V^{n}}{V^{m}} \\
d_{1} & =d_{C P_{i}, j}-\frac{d_{j, k}}{2} ; \quad d_{2}=d_{C P_{i}}+\frac{d_{j, k}}{2}
\end{aligned}
$$

where $d_{B W}^{m, n}$ is the length of the TBW associated aircraft pair $m, n$, and $d_{i}$ and $d_{f}$ are the initial and final distance from the critical section for the aircraft $n$. Lastly, the equations for the location of the TBWs are

$$
\begin{aligned}
\mathrm{TBW} & =\left[t_{0}^{n}-t_{b}, t_{0}^{n}-t_{a}\right] \\
t_{b} & =\frac{d_{4}}{V^{n}} ; t_{a}=\frac{d_{3}}{V^{n}} \\
d_{4} & =d_{C P_{i}, k}+\frac{d_{j, k}}{2}-d_{f} ; \quad d_{3}=d_{4}-d_{B W}^{m, n}
\end{aligned}
$$

where $t_{a}$ and $t_{b}$ are the time limits of the TBWs, i.e. the temporary limits that inhibit the introduction the aircraft $n$ depending on the velocities and entry times of the aircraft pair. To know the TBWs is crucial for the safety of further flight-plan elaboration of RPAS.

Developing the above equations, an expression is achieved that relates to the influence of the TBW's size with the velocity of the aircraft involved

$$
d_{B W}^{m, n} / V^{n}=t_{a}^{m, n}-t_{b}^{m, n}=d_{j, k}\left(\frac{1}{V^{m}}+\frac{1}{V^{n}}\right)
$$

The relation is inversely proportional with the airspace geometry and the velocity of both aircraft. If the 


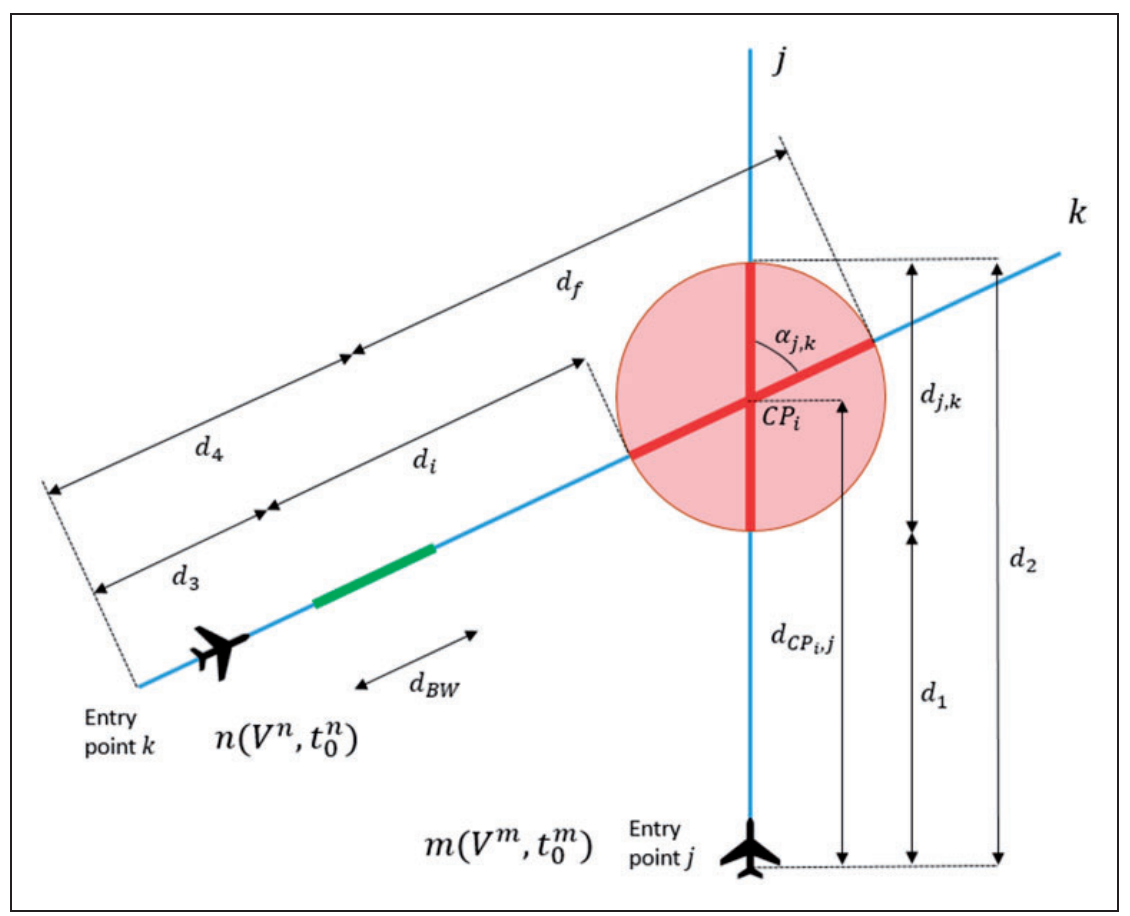

Figure 3. Representation of temporary-blocking window concept.

crossing angle decreases, the critical section and the TBW increase. The same concept applies to the velocity of both aircraft.

However, there is an exceptional case of study in which the RPAS flies the same airway of a conventional aircraft. Depending on the aircraft ahead (conventional aircraft or RPAS), different TBWs are obtained:

- If the conventional aircraft is ahead, it is enough to maintain current horizontal separation minima with the RPAS. The reason is that both aircraft enters fulfilling the horizontal separation minima, and the ahead conventional aircraft will increase the separation with the RPAS. Therefore, TBW is calculated as follows

$$
T B W=\left[t_{0}^{n} ; t_{0}^{n}+\frac{S_{\min }}{V^{c o n}}\right]
$$

- If the RPAS is ahead, it is required to ensure the separation minima throughout the airspace. In this case, the conventional aircraft will constantly reduce the separation with the RPAS. Then, TBW is calculated as follows

$$
\mathrm{TBW}=\left[t_{0}^{R P A S} ; t_{0}^{R P A S}+\frac{\left(L_{j}-S_{\min }\right)}{V^{R P A S}}-\frac{L_{j}}{V^{c o n}}\right]
$$

\section{Safety metrics}

The goal of the safety metrics is to assess the impact of RPAS integration regarding operational risk.
These safety metrics pretend to detect which airways favour their integration or which inhibit them. The safety metrics selected in this research are oriented for tactical planning in which the regulator disposes of operational information, i.e. a flight schedule. However, uncertainty associated with the flights (position and velocity) is not considered. Therefore, the authors have selected three safety metrics: number of conflicts, conflict severity and airway availability.

Number of conflicts $\left(N_{c}\right) . N_{c}$ is the number of times that the separation between an aircraft pair infringes the separation minima

$$
N_{c}=\text { number of aircraft pairs that } D^{m, n}<S_{\text {min }}
$$

The number of conflicts in tactical planning should be none. This implies that conflicts should happen only by non-planned circumstances.

Conflict severity $\left(\theta^{m, n}\right)$. Conflict severity is a safety indicator of the conflict seriousness because not every conflict implies the same impact. One conflict between an aircraft pair $m, n$ will be more serious as a function of their time duration $\left(\tau^{m, n}\right)$ and the minimum distance achieved $\left(d_{\text {min }}^{m, n}\right)$. Conflict severity is the combination of both parameters

$$
\theta^{m, n}=\tau^{m, n}\left(S_{\min }-d_{\min }^{m, n}\right)
$$

Figure 4 describes a conflict situation depending on the separation and the time. This situation represents 


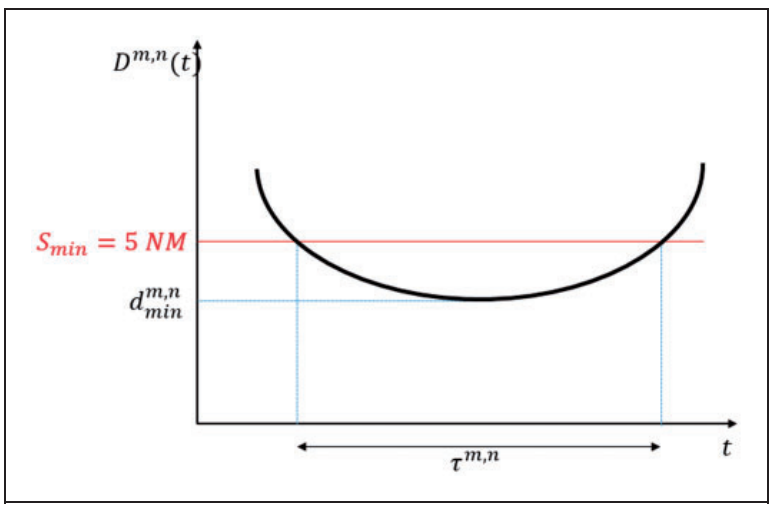

Figure 4. Evolution of a conflict between an aircraft pair.

a horizontal separation minima infringement characterised by the parameters $\tau^{m, n}$ and $d_{m i n}^{m, n}$.

Severity is used as a benchmarking value. Severity classifies a conflict depending on the time duration, and the minimum distance reached. This classification follows the concept of ESARR $4^{39}$ to manage the conflict resolution according to the severity of the conflicts.

Airway availability $\left(\lambda_{j}\right)$. This indicator aims to calculate the risk exposition of an aircraft flying an airway. This indicator is called airway availability because it links the time span the aircraft can safely fly an airway with the time span the aircraft can suffer a conflict. Knowing the airways that present higher availability (the time span the aircraft can safely fly without suffering a conflict), it can be extracted the airways that favour the integration of RPAS.

$\lambda_{j}$ is calculated by the size of overall TBW $d_{B W}^{m, n}$ that affect the airway $j$, calculated in the previous section. Therefore, the risk exposition of an aircraft relates the non-available time $\left(t_{N A_{j}}\right)$ and the exposition time $\left(t_{\text {exp }}\right)$

$$
\lambda_{j}=1-\frac{t_{N A_{j}}}{t_{\text {exp }}} \quad \text { where } \quad 0 \leqslant \lambda_{j} \leqslant 1
$$

where $t_{N A_{j}}=\sum_{m j} \frac{d_{B W}^{m, n}}{V^{m}}$

Herein, the exposition time relates to one-hour schedule. A minor TBW implies a bigger airway availability, which is beneficial to the risk exposition. Moreover, airway availability is a novel indicator defined in this work. There is no previous knowledge about the threshold that this indicator should acquire. Then, the authors propose a division into four stretches $(0-25 \%, 25-50 \%, 50-75 \%$ and $75-100 \%)$. Airways with airway availability greater than $50 \%$ are airways where RPAS should be included.

\section{Case study}

This section characterises the static and dynamic variables identified in section Base scenario that underlies the base scenario. Moreover, it describes the flight schedule for conventional aircraft where the introduction of RPAS will be assessed.

\section{Base scenario}

Once we have described the methodology, the airspace volume that we have selected to apply the methodology is LECMZGZ. This airspace is located in the upper airspace of Spain. A high rate of cruise flights and the scarce influence of climbing/descending flights characterise it. The methodology is developed for every FL that constitutes the airspace volume; however, it has been particularised for the FL 300. Conventional air traffic at FL 300 is reduced compared with upper FLs. Fourteen airways and 30 crossing points constitute LECMZGZ geometry, see Figure 5.

Air traffic flows have clear features for the corridor North-South (37.7\%) and East-West (39.5\%). The percentage of conventional aircraft climbing or descending represent less than $15 \%$, and their influence is discarded in this approach. Table 1 provides the crossing angles between airways of LECMZGZ. The symbol "." denotes airway pairs that do not intersect in LECMZGZ. Attending to Table 1, LECMZGZ airspace presents an advantage because the majority of the crossing points only intersects between two airways.

\section{Flight schedule for conventional aircraft}

Table 2 provides the flight schedule of conventional aircraft of 26 July 2017. This is a one-hour schedule constituted by four conventional aircraft flying the FL 300 , which represent the maximum air traffic demand.

Conventional aircraft only uses three airways; two aircraft use one airway. These three airways intersect with the rest of the airways, which imply restrictions for the calculation of safety indicators. The velocity of the aircraft varies from 400 to 500 knots (kts). Figure 6 represents the conceptual flight schedule of conventional aircraft.

Table 2 provides the flight schedule of conventional aircraft of 26 July 2018. This is a one-hour schedule constituted by four conventional aircraft flying the FL 300 , which represent the maximum air traffic demand.

\section{Results}

This section provides the results obtained by the introduction of RPAS in the previous schedule. The introduction of RPAS is detailed for the airways UN869 and UN871, although the results for the RPAS introduction through every airway of the airspace are presented later on. These cases represent the two operational situations that can appear: (1) Airway UN869 does not have air traffic and present two intersections with operated airways (UM601 and UN725), and (2) Airway UN871 is initially operated by a 


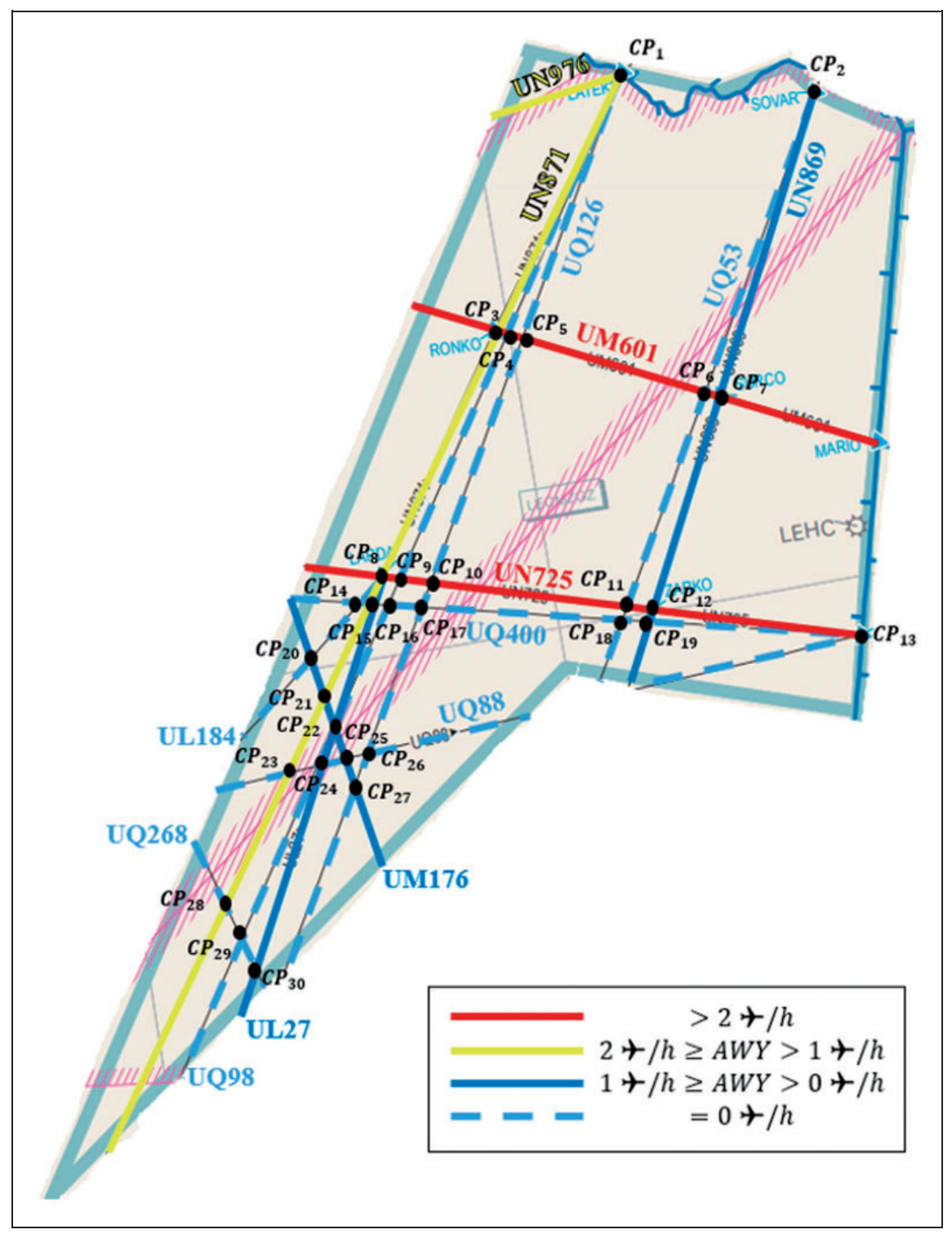

Figure 5. Scheme of LECMZGZ airspace.

Table I. Crossing angles $\left({ }^{\circ}\right.$ ) between airways in LECMZGZ.

\begin{tabular}{|c|c|c|c|c|c|c|c|c|c|c|c|c|c|c|}
\hline \multirow{2}{*}{$\frac{\text { Airway (j) }}{\alpha_{j, k}}$} & \multicolumn{14}{|c|}{ Airway $(k)$} \\
\hline & UM60I & UN725 & UQ400 & UQ88 & UN976 & ULI84 & UQ268 & UL27 & UN87I & UQ98 & UQI26 & UQ53 & UN869 & UMI76 \\
\hline UM60I & - & - & - & - & - & - & - & - & 80 & 82 & 85 & 85 & 88 & - \\
\hline UN725 & - & - & 4 & 21 & - & 57 & - & 79 & 71 & 73 & 76 & 76 & 79 & - \\
\hline UQ400 & - & 4 & - & 17 & - & 53 & - & 75 & 68 & 69 & 72 & 72 & 75 & - \\
\hline UQ88 & - & 21 & 17 & - & - & - & - & 58 & 51 & 52 & 55 & - & - & 85 \\
\hline UN976 & - & - & - & - & - & - & - & - & 44 & 46 & 49 & - & - & - \\
\hline ULI84 & - & 57 & 53 & - & - & - & - & 22 & 15 & - & - & - & - & 59 \\
\hline UQ268 & - & - & - & - & - & - & - & 44 & 51 & 50 & - & - & - & - \\
\hline UL27 & - & 79 & 75 & 58 & - & 22 & 44 & - & 7 & 6 & - & - & - & 37 \\
\hline UN87I & 80 & 71 & 68 & 51 & 44 & 15 & 51 & 7 & - & 2 & 5 & - & - & 44 \\
\hline UQ98 & 82 & 73 & 69 & 52 & 46 & - & 50 & 6 & 2 & - & 3 & - & - & 43 \\
\hline UQI26 & 85 & 76 & 72 & 55 & 49 & - & - & - & 5 & 3 & - & - & - & 40 \\
\hline UQ53 & 85 & 76 & 72 & - & - & - & - & - & - & - & - & - & 3 & - \\
\hline UN869 & 88 & 79 & 75 & - & - & - & - & - & - & - & - & 3 & - & - \\
\hline UMI76 & - & - & - & 85 & - & 59 & - & 37 & 44 & 43 & 40 & - & - & - \\
\hline
\end{tabular}


conventional aircraft. The entry time of the RPAS does not vary at any simulation. The airway UN869 does not have conventional aircraft while the airway UN871 does. The calculation of the three safety indicators permits to detect the number of conflicts, to measure the conflict severity and to calculate the airway availability. By the calculation of the different indicators, the methodology provides the airways that

Table 2. Flight schedule of conventional aircraft.

\begin{tabular}{lllll}
\hline Aircraft & Airway & $t_{0}^{m}$ & $\mathrm{FL}$ & $V^{m}(\mathrm{kts})$ \\
\hline 1 & UM60I & $00: 12: 48$ & 300 & 431 \\
2 & UM60I & $00: 22: 30$ & 300 & 500 \\
3 & UN725 & $00: 31: 15$ & 300 & 411 \\
4 & UN87I & $00: 36: 21$ & 300 & 436 \\
\hline
\end{tabular}

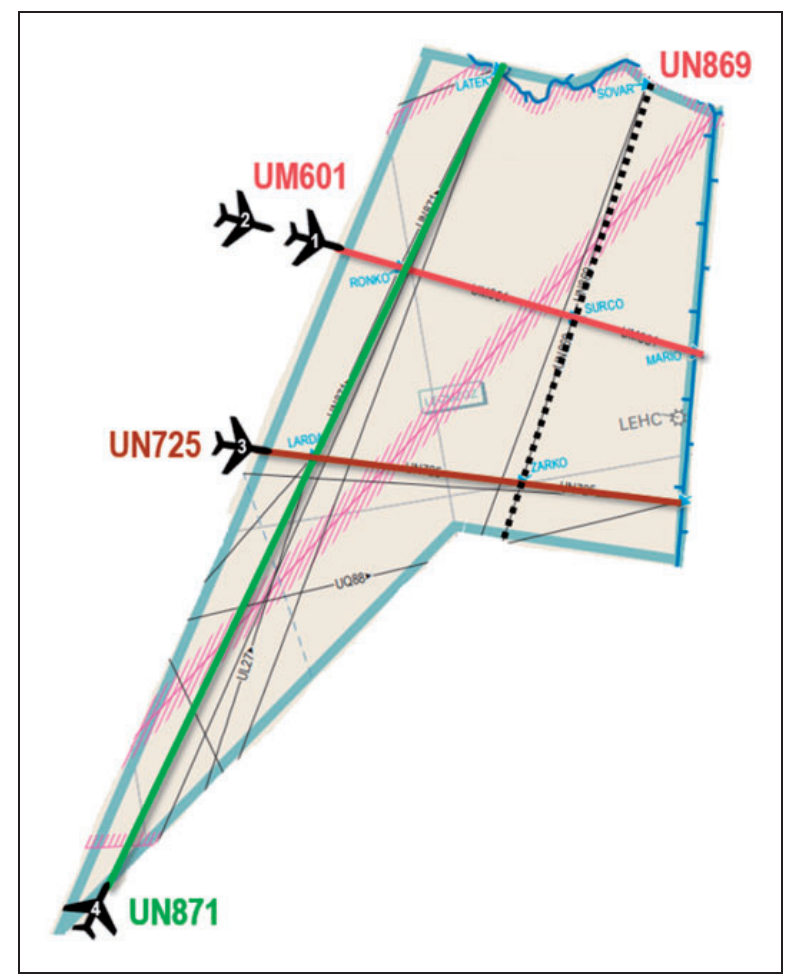

Figure 6. LECMZGZ airspace and airways involved in the simulations. favour the integration of RPAS and the airways that inhibit them.

The TBWs are calculated for every aircraft pair, i.e. the time span that the airways are blocked because a separation minima infringement will occur. The primary features of the TBWs are:

- The time duration of the TBWs depends on the crossing angle of the airways and the ground speed of the aircraft involved, and

- The time location of the TBWs depends on the entry time of the conventional aircraft and RPAS, length of the airways, the ground speed and the distance between the airway entry-point and the crossing point.

\section{RPAS introduction through airway UN869}

One RPAS is assumed to operate through the airway UN869 with a specific entry time $\left(t_{0}^{R P A S}=0: 23: 20\right.$ or $\left.1700 \mathrm{sec}\right)$. The RPAS fly at the same FL than conventional aircraft and the ground speed is $250 \mathrm{kts}$. Table 3 shows the results obtained for the TBWs and the airway availability indicators.

Table 4 shows the temporary location of the TBWs for every aircraft. In the case we change the entry time, the location of the TBWs will vary, but the size of them will not vary because they only depend on the geometrical and dynamic features.

The simulation does not provide any conflict between the RPAS and the conventional aircraft.

Regarding the temporary-blocking windows: a conventional aircraft generates a TBW bigger when the velocity of the involved aircraft is reduced. As explained in section 2.4, the TBW generated by an aircraft over another aircraft is minor than the TBW generated by a conventional aircraft over the RPAS. In this way, the most restrictive case would be the TBW between two RPAS. Then, the ANSP should allocate the RPAS operation through non-intersecting airways.

Regarding the airway availability $\left(\lambda_{j}\right)$, the airway UM601 is only affected by the case of one conventional aircraft because both aircraft does not run into the airway at the same time. When aircraft 2 pierces into the sector, aircraft 1 has already left it.

Table 3. Time duration (sec) of TBWs and airway availability indicator.

\begin{tabular}{lllllll}
\hline Airway $(j)$ & Aircraft I & Aircraft 2 & Aircraft 3 & Aircraft 4 & RPAS & $\lambda_{j}$ \\
\hline UM60I & - & - & - & 166 & 212 & 0.8628 \\
UM60I & - & - & - & 171 & 216 & \\
UN725 & - & - & - & 186 & 236 & 0.9055 \\
UN87I & 153 & 157 & 180 & - & - & 0.8511 \\
UN869 (RPAS) & 212 & 216 & 236 & - & - & 0.6472 \\
\hline
\end{tabular}


Table 4. Time location (sec) of the TBWs.

\begin{tabular}{llllll}
\hline Airway $(j)$ & Aircraft I & Aircraft 2 & Aircraft 3 & Aircraft 4 & RPAS \\
\hline UM60I & - & - & - & {$[11 ; 177]$} & {$[423 ; 635]$} \\
UM60I & - & - & - & {$[593 ; 764]$} & {$[1015 ; 1231]$} \\
UN725 & - & - & - & {$[1339 ; 1525]$} & {$[1289 ; 1525]$} \\
UN87I & {$[2772 ; 2925]$} & {$[2767 ; 2924]$} & {$[2531 ; 27 \mid 1]$} & - & - \\
UN869 (RPAS) & {$[1833 ; 2045]$} & {$[1819 ; 2035]$} & {$[2050 ; 2286]$} & - & - \\
$t_{0}$ & 768 & 1350 & 1875 & 2181 & 1700 \\
\hline
\end{tabular}

Therefore, the total airway occupancy is the addition of the airway occupancy by each aircraft but independently. Consequently, the indicator $\lambda_{j}$ depends directly on both the aircraft velocity and the airway length. The bigger the occupancy of each airway, the lesser the airway availability. This fact implies that the airway availability in which the RPAS flies (UN869) is the lowest. In other words, while $\lambda_{U N 869(R P A S)}=0,6472, \lambda_{j}$ for the rest of airways are between 0.85 and 0.9 .

This result shows the high impact that the introduction of RPAS implies compared with the conventional aircraft: the RPAS impact provides a blocking in the airspace around three times the impact due to a conventional aircraft. Moreover, the values of airway availability for the airway UM601 and UN871 are very similar, although in the airway UM601 two aircraft operates. This means that the airway geometry entails a significant impact and can impose a bigger restriction than the number of aircraft.

\section{RPAS introduction through airway UN87I (with conventional traffic)}

One RPAS flies through the airway UN871, which initially has one conventional aircraft. The RPAS goes ahead of the conventional aircraft with a time separation of $481 \mathrm{~s}$. The operational parameters do not vary from the previous simulation. Table 5 shows the results obtained for TBWs and the airway availability indicators.

In this simulation does not appear any conflict and, therefore, the number of conflicts is null as well as the conflict severity.

The TBW generated by the conventional aircraft over the RPAS is only 41 seconds. This means that, if an RPAS pierces into the airspace 42 seconds after the conventional aircraft 4 , a conflict will not occur. Conversely, if the RPAS is ahead of the conventional aircraft, the TBW generated increases its time size to $738 \mathrm{~s}$ (18 times bigger). Then, when an RPAS would operate a large airway (e.g. $400 \mathrm{NM}$ ), the ANSP could require the airway segregation for RPAS to avoid unsafe situations.

Regarding the indicator $\lambda_{j}$, the airways UM601 and UN725 keep the same previous value because no modifications appear in the schedule. However, the airway UN871 shows a reduction of the airway availability fewer than half by the RPAS introduction $(49 \%)$. In this case, the RPAS should not operate the airway UN871 in this 1-h schedule because $\lambda_{j}$ is minor than $50 \%$.

\section{Discussion of results}

The same process described in the previous section was performed for every airway of the airspace. The aim was to analyse the safety indicators obtained by the RPAS introduction through every airway. Table 6 shows $\lambda_{j}$ results sorted in descending order.

The above values were calculated by the same operational conditions of the conventional aircraft and RPAS. According to the airway indicator, airways were divided into four groups:

- The most suitable and available airways were UN976, UQ268 and UM176 because the introduction of one RPAS implied a similar impact like one conventional aircraft (around 10-15\%).

- The airways UQ88, UN869 and UQ53 reduced the airway availability up to $65 \%$. This means that these airways could be used for the integration of RPAS although they were barely recommended. The integration of RPAS through these airways would require further analysis detailing the schedule and the operational features in order to validate the RPAS introduction.

- The airways UL184 and UL27 provided inefficient results because they reduced the airway availability up to $25 \%$ in the case an RPAS operates through them, i.e. it barely could be introduced a conventional aircraft and its operation could be segregated for an RPAS.

- Finally, the airways UQ126, UQ400 and UQ98 provided a full restriction of their use for RPAS. Therefore, they should be forbidden for RPAS. The main reason was these airways intersected with other airways operated by conventional aircraft as UN871 and UN725 with a crossing angle lesser than $5^{\circ}$. In these cases, airways should not be considered as independent airways but a coincident airway.

Figure 7 shows the physical results obtained by previous calculations. The main limitation is that the 
Table 5. Time duration (sec) of TBWs and airway availability indicator.

\begin{tabular}{lllllll}
\hline Airway & Aircraft I & Aircraft 2 & Aircraft 3 & Aircraft 4 & RPAS & $\lambda_{j}$ \\
\hline UM60I & - & - & - & 166 & 229 & 0.8626 \\
UM60I & - & - & - & 171 & 234 & \\
UN725 & - & - & - & 186 & 251 & 0.9050 \\
UN87I & 153 & 157 & 180 & - & 41 & 0.4962 \\
UN87I (RPAS) & 215 & 219 & 244 & 738 & - & \\
\hline
\end{tabular}

Table 6. $\lambda_{j}$ for every airway in LECMZGZ.

\begin{tabular}{|c|c|c|c|c|c|c|c|c|c|c|c|c|c|c|}
\hline Airway (RPAS) & UN976 & UQ268 & UMI76 & UN725 & UM60I & UQ88 & UN869 & UQ53 & UN87I & ULI84 & UL27 & UQ126 & UQ400 & UQ98 \\
\hline & 0.921 & 0.854 & 0.840 & 0.825 & 0.795 & 0.726 & 0.647 & 0.645 & 0.496 & 0.413 & 0.248 & 0.016 & 0 & 0 \\
\hline
\end{tabular}

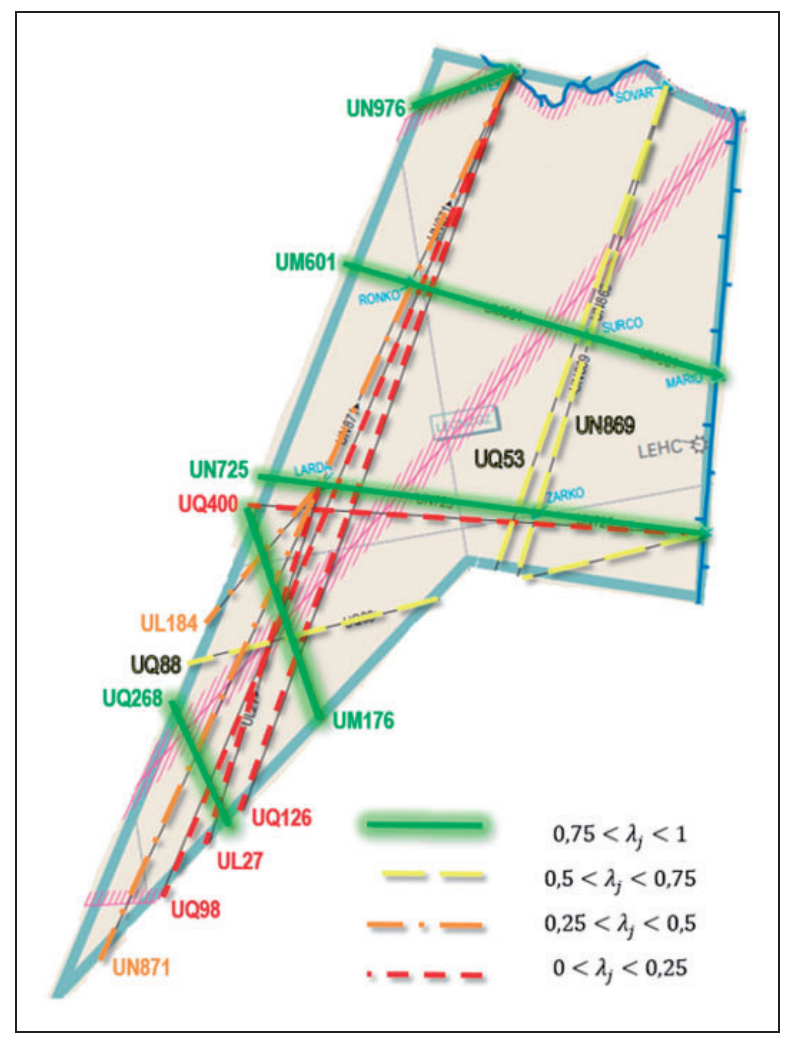

Figure 7. $\lambda_{j}$ values depending on the airway it is introduced the RPAS.

airways that favour the introduction of RPAS are secondary airways of this airspace. Nonetheless, it is evident that in the case of an RPAS operation from North to South (or vice versa), it should fly the airways UQ83 or UN869. In the case of an RPAS operation from East to South (or vice versa), it should select the airways UM601, UN725 or UQ88. However, UM601 and UN725 dispose of conventional aircraft, and the RPAS introduction should be timely segregated so as not to generate any conflict.

Regarding the other safety indicators, Table 7 provides the results of the conflicts that arose depending
Table 7. Severity indicator for different RPAS operation.

\begin{tabular}{llllr}
\hline Airway $_{\text {RPAS }}$ & Airway $_{\text {Con }}$ & $d_{\min }^{m, n}(\mathrm{NM})$ & $\tau^{m, n}(\mathrm{~s})$ & $\theta^{m, n}$ \\
\hline UQ98 & UN87I & 0.4969 & 193 & 869.1 \\
UQ126 & UN725 & $1.038 I$ & 82 & 324.9 \\
UMI76 & UN87I & 0.9564 & 55 & 222.4 \\
UQ400 & UN87I & 3.1431 & 67 & 124.4 \\
UN976 & UN87I & 3.6134 & 39 & 54.1 \\
\hline
\end{tabular}

on the airway operated by the RPAS. These results are sorted in descending order of severity.

The maximum number of conflicts was 1 for each simulation and occurred in five airways (from 14). The results showed in Table 7 were the airways of the RPAS and the conventional aircraft, the minimum distance between both aircraft, the time size of the conflict and the indicator of conflict severity. The emphasis was significant, as the time entry of the RPAS did not affect the calculation of the airway indicator, the entry time directly influenced the conflict severity. Then, the exposure time and the minimum distance highly varied with the entry time. Therefore, the values provided by the indicator $\theta$ were obtained for a particular schedule. The results for the different conflicts were:

- The first conclusion was that almost every conflict was produced with the conventional aircraft of the airway UN871. This airway had the bigger number of intersections.

- The worst conflict was generated when the RPAS operated through the closest airway (UQ98) to the conventional aircraft 4 (UN871). In this schedule, the conflict severity was huge $\theta^{m, n}=869.1$. In this case, the minimum distance achieved was $0.5 \mathrm{NM}$ and the conflict duration of $193 \mathrm{~s}$. The reason was the airway UQ98 intersected with the airway UN871 with a crossing angle of $2^{\circ}$. Therefore, both airways behaved as a singular airway. This result is significant because it implies that the 
airways that intersect with a crossing angle very small $\left(<10^{\circ}\right)$ they should not be considered as independent airways but as a coincident airway. These results confirmed the conclusions from previous research..$^{25,28}$

- The conflict between the airways UQ126 and UM176 reached a minimum distance of $1 \mathrm{NM}$; however, the time duration halved the above conflict. The exposure time of the above conflict was significantly bigger because the critical section was larger. However, it was only 41 and 26 seconds since both aircraft infringed the separation minima until the minimum distance was reached. In contrast, the above conflict required $96 \mathrm{~s}$. Then, the gradient of the separation reduction was reduced faster at these conflicts. It means that, once the separation minima infringement occurs, the ATC has lesser time to deal with it.

- Finally, the airway UN976 was detected as the most available airway, and it had the minor conflict of them. The reduced length of UN976, the scarce number of intersections and a beneficial geometry provided a conflict with reduced severity.

Therefore, this research allowed for applying the safety metrics for tactical planning explained above for the RPAS integration: These safety metrics calculated the number of conflicts, the conflict severity and the airway availability as a function of 1-h schedule. Moreover, this methodology determined temporary restrictions for the integration of RPAS in non-segregated airspace. Although the results obtained were remarkable for the development of the RPAS integration jointly with conventional aircraft, it is required to deepen in two crucial aspects in further work.

The first need is the implementation of this tactical analysis in a whole process of the RPAS flight plan. This means that the operator should modify the RPAS flight plan depending on the time-dependent airway availability and, in the future, the ANSP should modify the flight plan depending on the temporary restrictions. The second need is that these safety metrics must be considered as a preliminary step to the establishment of a target level of safety, which allows defining limitations for the number of RPAS that can be introduced in non-segregated airspace. In this way, it could be achieved an equilibrium between the number of RPAS, the number of conventional aircraft and the risk accepted.

\section{Conclusions}

The integration of RPAS in non-segregated airspace is one of the most complex challenges of civil aviation. This research tried to bring to light some of the concerns that the aviation community have to deal with. Mainly, this paper focused on the implementation of safety metrics for tactical planning to assess the integration of RPAS jointly with conventional aircraft.
The methodology was divided into five modules. The base scenario described the geometrical and operational information of airspace. The path modelling generated the trajectories of the aircraft based on the operational features of a schedule. The conflict detection module evaluated the distance between aircraft pairs to detect any separation minima infringement and characterised the minimum distance and the time span of the conflict. The TBWs calculated the temporary restrictions that were imposed according to the airways operated by intersecting aircraft. Finally, the safety indicators appraised the viability of the integration of RPAS through the number of conflicts, the conflict severity and the airway availability.

This methodology provided a step forward on the integration of RPAS. In terms of the TBWs, the methodology calculated the temporary restrictions that limit the operation of an RPAS flight plan. If the RPAS flies ahead of a conventional aircraft in the same airway, the size of the TBWs is 18 times bigger than to the contrary. Regarding the airway availability indicator, it determined the airways that should be operated by RPAS. They were divided into four groups depending on the airway availability for RPAS. Particularly, the integration of RPAS imposed a decrease in airway availability three times bigger than conventional aircraft. Conflict severity is a time-dependent indicator that highly depends on the 1-h schedule. Thus, no overall conclusions can be extracted about it. However, it provided information about the prioritisation process to solve conflicts. Further work should focus on the implementation of these tactical results in a whole flight plan process and the application of the 4D trajectory concept to the TBWs. The goal must be to provide geographical and temporary limitations to the operation of RPAS in order to ensure safe operations in non-segregated airspace.

\section{Acknowledgements}

This Project has been developed under the OIDATM (Observatory for the Advancement of Air Traffic Management) promoted by ISDEFE. Particularly, the authors would like to acknowledge Miguel A. Martín Blanco, Maria Anta Garcia and Susana Duran Vizuete.

\section{Declaration of Conflicting Interests}

The author(s) declared no potential conflicts of interest with respect to the research, authorship, and/or publication of this article.

\section{Funding}

The author(s) disclosed receipt of the following financial support for the research, authorship, and/or publication of this article: This paper was funded by the project "Cátedra ISDEFE sobre Gestión del Tránsito Aéreo (ATM)" with reference number CAT1814150078 at the Universidad Politécnica de Madrid. 


\section{ORCID iD}

Javier A Pérez-Castán (D) https://orcid.org/0000-0002-01129792

Álvaro Rodriguez-Sanz (D) https://orcid.org/0000-0002$1555-3914$

\section{References}

1. European RPAS Steering Group. Roadmap for the integration of civil remotely-piloted aircraft systems into the European Aviation System. June 2013.

2. EASA. Concept of operations for drones: a risk-based approach to regulation of unmanned aircraft. May 2015.

3. EASA. NPA 2017-05 (B): introduction of a regulatory framework for the operation of drones. 2017.

4. Civil Aviation Safety Authority and Australian Government, "Review of aviation safety regulation of remotely piloted aircraft systems," 2018.

5. Dalamagkidis K, Valavanis KP and Piegl LA. On unmanned aircraft systems issues, challenges and operational restrictions preventing integration into the National Airspace System. Prog Aerosp Sci 2008; 44: 503-519.

6. Netjasov F and Janic M. A review of research on risk and safety modelling in civil aviation. J Air Transp Manag 2008; 14: 213-220.

7. Pérez-Batlle M, Pastor E and Prats X. Evaluation of separation strategies for unmanned aerial systems. In: Proceedings of the 5th international congress on research in air transportation, Berkeley, USA, 24 May 2016, pp.1-8.

8. Pastor E, Royo P, Cuadrado R, et al. On the design of UAS horizontal separation maneuvers. In: SESAR innovation days, Braunschweig, Germany, 27-29 November 2012.

9. Zarandy A, Zsedrovits T, Pencz B, et al. A novel algorithm for distant aircraft detection. In: International conference on unmanned aircraft systems (ICUAS), 2015, pp.774-783.

10. Lin $Y$ and Saripalli S. Collision avoidance for UAVs using reachable sets. In: International conference on unmanned aircraft systems (ICUAS), Denver, Colorado, 9-12 June 2015, pp.226-235.

11. Pierpaoli $\mathrm{P}$ and Rahmani A. UAV collision avoidance exploitation for noncooperative trajectory modification. Aerosp Sci Technol 2017; 1: 1-11.

12. Pérez-Castán JA, Comendador FG, Rodríguez-Sanz Á, et al. Conflict-resolution algorithms for RPAS in nonsegregated airspace. Aircr Eng Aerosp Technol 2019; 91: 366-372.

13. Clothier R, Walker R, Fulton N, et al. A casualty risk analysis for unmanned aerial system (UAS) operations over inhabited areas. In: Second Australasian unmanned air vehicle conference, Melbourne, Australia, 19-22 March 2007.

14. Evans AW, Foot PB, Mason SM, et al. Third party risk near airports and public safety zone policy. Res Dev Dir Natl Air Traffic Serv Limited, R\&D Rep. 9636, 1997. RDD File Reference 8CS/091/03/10.

15. Weibel R and Hansman RJ. Safety considerations for operation of different classes of UAVs in the NAS. In: AIAA 3rd "Unmanned Unlimited" technical conference, workshop and exhibit, Chicago, USA, 20-23 September 2004, September, p.11.
16. Melnyk R, Schrage D, Volovoi V, et al. A third-party casualty risk model for unmanned aircraft system operations. Reliab Eng Syst Saf 2014; 124: 105-116.

17. Lum $\mathrm{C}$ and Waggoner B. A risk based paradigm and model for unmanned aerial systems in the national airspace. In: AIAA Infotech@Aerospace conference, St. Louis, USA, 29-31 March 2011, pp.1-31.

18. Román-Cordón R, Arnaldo-Valdés R-M, CuernoRejado $\mathrm{C}$, et al. Integración de aeronaves pilotadas por control remoto en espacio aereo : diseño y desarrollo del interfaz de la estación de control en tierra. DYNA 2017; 93.

19. Clothier RA, Palmer JL, Walker RA, et al. Definition of an airworthiness certification framework for civil unmanned aircraft systems. Saf Sci 2011; 49: 871-885.

20. Clothier R, Clothier RA, Williams BP, et al. Challenges to the development of an airworthiness regulatory framework for unmanned aircraft systems challenges to the development of an airworthiness regulatory framework for unmanned aircraft systems describing the risk paradigm. In: 16th Australian aerospace congress, 2015, pp.87-98.

21. Clothier RA, Williams BP and Fulton NL. Structuring the safety case for unmanned aircraft system operations in non-segregated airspace. Saf Sci 2015; 79: 213-228.

22. Weibel R and Hansman RJ. Safety considerations for operation of different classes of UAVs in the NAS. In: AIAA 3rd "Unmanned Unlimited" technical conference work and exhibit 2004; September, pp.1-11, 2004.

23. JARUS. Specific Operations Risk Assessment (SORA) - D.04 - v2.0. 2019.

24. EASA. NPA 2017-05 (A): Introduction of a regulatory framework for the operation of drones. 2017.

25. Netjasov F. Framework for airspace planning and design based on conflict risk assessment Part 1 : Conflict risk assessment model for airspace strategic planning. Transp Res Part C Emerg Technol 2012; 24: 190-212.

26. Geisinger KE. Airspace conflict equations. Transp Sci 1985; 19: 139-153.

27. Mehadhebi K and Lazaud P. A synthesis of current collision risk models. In: ICAO separation and airspace safety panel, 5th meeting of the working group of the whole (SASP-WG/WHL/5-WP/9), 2004.

28. Pérez-Castán JA, Gómez Comendador F, RodríguezSanz A, et al. RPAS conflict-risk assessment in nonsegregated airspace. Saf Sci 2019; 111: 7-16.

29. Kim SH. Conflict risk assessment of structured and unstructured traffic of small unmanned aircraft systems. In: 2018 Aviation technology integration operations conference, 15, Atlanta, USA, 25-29 June 2018, pp.2529, 2018.

30. Jenie YI, Van Kampen E-J, Ellerbroek J, et al. Safety assessment of unmanned aerial vehicle operations in an integrated airspace. In: AIAAInfotech@Aerospace, San Diego, USA, 4-8 January 2016, pp.1-11.

31. Sunil E, Ellerbroek J, Hoekstra JM, et al. Three-dimensional conflict count models for unstructured and layered airspace designs. Transp Res Part C Emerg Technol 2018; 95: 295-319.

32. Tang H, Robinson JE and Denery DG. Tactical conflict detection in terminal airspace. J Guid Control Dyn 2011; 34: 403-413.

33. Paielli RA and Erzberger H. Trajectory specification for terminal air traffic : pairwise conflict detection 
and resolution. In: 17th AIAA aviation technology, integration, and operations conference, Denver, USA, 5-9 June 2017, pp.1-19.

34. ENAIRE. ENR 1.6-1 ATS surveillance services and procedures. 2016.

35. Speijker LJP, et al. RASMAR final report: risk analysis of simultaneous missed approaches on Schiphol converging runways I9R and 22. 2001.

36. Henry M, Schmitz S, Revenko N, et al. A Monte Carlo simulation for evaluating airborne collision risk in intersecting runways. In: AIAA modeling and simulation technologies (MST) conference, Boston, USA, 19-22 August, 2013.

37. Pérez-castán JA, Comendador FG, Arnaldo RM, et al. Conflict resolution algorithms for the integration of continuous climb operations in a Terminal Maneuver Area. In: 30th Congress of the International Council of Aeronautical Sciences, Daejeon, Korea, 25-30 September 2016, p.10.

38. Pérez-Castán JA, Gómez Comendador F, RodríguezSanz A, et al. Impact of continuous climb operations on airport capacity. Transp Res Part C Emerg Technol 2018; 96: 231-250.

39. EUROCONTROL. ESARR 4: risk assessment and mitigation in ATM. Eurocontrol Saf Regul Requir 2001; 1-22.

\section{Appendix}

\section{Notation}

$\alpha_{j, k}$
$C O N V / R P A S$
$C P_{i}$
$d_{B W}^{m, n}$

aircraft/RPAS indicator

length of the temporary-blocking window for the aircraft pair $m, n$ $d_{e}, d_{f}$

$d_{j, k}$

$d_{\text {min }}^{m, n}$

$D(t)$

$\theta^{m, n}$

$j$

$\lambda_{j}$

$L_{j}$

$N_{c}$

$S_{\text {min }}$

$t$

$t_{0}^{m}$

$t_{a}, t_{b}$

$t_{\text {exp }}$

$t_{N A_{j}}$

$\tau^{m, n}$

$V^{m}\left(V_{x}^{m}, V_{y}^{m}\right)$

$W\left(W_{x}, W_{y}\right)$

$X^{m}, Y^{m}, Z^{m}(t)$ distance from the entry point of the aircraft $m$ to the crossing point $C P_{i}$

Initial and final distance from the critical section for the aircraft $n$ conflict area between airways $j$ and $k$

minimum distance achieved between the aircraft pair $m, n$ separation between an aircraft pair depending on time conflict severity between an aircraft pair $m, n$ airway indicator ratio of time the airway $j$ is blocked airway Length number of conflicts horizontal separation minimum time entry time to the airspace of aircraft $m$ time limits of the temporaryblocking window exposition time non-available time of airway $j$ time duration of a conflict between the aircraft pair $m, n$ ground speed of aircraft $m$ wind trajectory coordinates of aircraft $m$ 